\title{
Civilisations
}

Revue internationale d'anthropologie et de sciences

humaines

58-1| 2009

American Afrocentrism(e)s américains

\section{Afrocentrismes américains}

Histoire, nationalisme noir et pratiques sociales

Pauline GUEDJ

\section{(2) OpenEdition}

\section{Journals}

Édition électronique

URL : http://journals.openedition.org/civilisations/2663

DOI : 10.4000/civilisations. 2663

ISSN : 2032-0442

Éditeur

Institut de sociologie de l'Université Libre de Bruxelles

\section{Édition imprimée}

Date de publication : 31 août 2009

Pagination : $9-21$

ISBN : 2-87263-026-0

ISSN : 0009-8140

\section{Référence électronique}

Pauline GUEDJ, « Afrocentrismes américains », Civilisations [En ligne], 58-1 | 2009, mis en ligne le 31 août 2009, consulté le 19 avril 2019. URL : http://journals.openedition.org/civilisations/2663 ; DOI : 10.4000 /civilisations. 2663

(c) Tous droits réservés 


\title{
Afrocentrismes américains \\ Histoire, nationalisme noir et pratiques sociales
}

\author{
Pauline GUEDJ
}

\section{Débats et controverses}

$\mathrm{E}$ 2000, paraissait le premier ouvrage de référence en langue française sur l'Afrocentrisme. Rassemblant des contributions rédigées par des spécialistes de plusieurs disciplines et consacrées à diverses aires géographiques et périodes historiques, Afrocentrismes, L'histoire des Africains entre Egypte et Amérique se penchait sur les multiples facettes de ce courant de pensée. Pour ce faire, les éditeurs de cet ouvrage, François-Xavier Fauvelle-Aymar, Jean-Pierre Chrétien et Claude-Hélène Perrot, entendaient s'appuyer sur le « mode de narration » de l' « historiographie critique » (2000 : 11), revenir sur les présupposés des écrits fondateurs de l'Afrocentrisme, et surtout, fournir aux lecteurs les clés d'un débat populaire et problématique qui opposeraient partisans d'une historiographie classique et « eurocentrée » et Afrocentristes, révolutionnaires, luttant corps et âme contre un africanisme colonial. Appelant, à l'encontre des Afrocentristes, à la rigueur scientifique et déplorant le pouvoir symbolique d'ouvrages emplis de "fausses théories ", les éditeurs se défendaient toutefois de toute intention polémique. "Avec honnêteté », ils clamaient leur respect pour l'Afrique, terrain d'étude victime de ses descriptions hâtives, et continent, méritant au même titre que l'Europe, d'être l'objet d'approches scientifiques finement argumentées et non idéologiques (2000: 10).

Toutefois, malgré les précautions prises par les éditeurs dans leur introduction, l'ouvrage ne manquait pas, dans certains de ses articles, de glisser vers l'accusation et d'opter pour un ton condescendant. Ainsi, sous la plume de Clarence Walker, Molefi Asante (voir Asante 1987, 1988, 1990) devenait un intellectuel au propos " douteux » (Walker $2000: 67)$, un penseur non « original » (2000: 70) dont « le succès parmi les Américains noirs peut être attribué au fait que, à l'heure actuelle, la pensée critique n'est pas en grande estime dans la communauté noire aux Etats-Unis » (2000 : 70-71). Ivan Van Sertima était dépeint par Bernard R. Ortiz de Montellano, comme un auteur « aux sources douteuses et obsolètes » dont les écrits n'intéressent que des « Afro-Américains prédisposés à accepter son message » (Ortiz de Montellano 2000 : 264-265). 
Ces critiques ${ }^{1}$ adressées souvent autant aux auteurs afrocentristes qu'à leur lectorat de prédilection, des Afro-Américains jugés crédules, constituent sans aucun doute l'écueil de l'ouvrage. Cette limite éveilla par ailleurs les foudres des penseurs afrocentristes francophones qui n'avaient déjà de cesse d'alimenter la polémique. Ainsi, en 2001, l'intellectuel congolais Théophile Obenga, disciple de Cheikh Anta Diop et collaborateur de Molefi Asante, rétorquait avec la publication d'un ouvrage, cette fois-ci ouvertement polémique, dans lequel Fauvelle-Aymar, Chrétien et Perrot étaient qualifiés de penseurs « racistes », dont l'approche paternaliste consisterait, sous couvert de respect pour le continent africain, à dénigrer le génie du peuple noir, ses accomplissements culturels et son lien généalogique avec l'Antiquité égyptienne. Conçu comme le manifeste d'une lutte « contre l'africanisme raciste, ancien ou moderne, colonial ou post-colonial » (Obenga 2001 : 7), le texte d'Obenga multipliait les attaques personnelles et injurieuses.

Bien qu'accusatrice et polémique, la tonalité du débat opposant Fauvelle-Aymar, Chrétien et Perrot à Obenga n'était pas, il y a huit ans déjà, une nouveauté. Aux EtatsUnis, les confrontations entre Afrocentristes et anti-Afrocentristes s'étaient imposées dans les universités depuis la fin des années quatre-vingt-dix et s'étaient cristallisées autour de la publication d'un ouvrage jugé par certains provocateur : Not out of Africa. How Afrocentrism became an excuse to teach myth as history (1996). Dans cet ouvrage, l'historienne Mary Lefkowitz s'insurgeait à la fois contre le texte de Martin Bernal Black Athena (1987), texte-phare de l'Afrocentrisme et contre la pratique dans certaines universités américaines d'enseignements se réclamant de ce courant de pensée.

Comme en France dans le cas de la controverse précédemment citée, la publication de l'ouvrage de Lefkowitz fut suivie d'une polémique ; certains auteurs afrocentristes, comme Maulana Karenga, se confrontant à l'historienne dans des débats publics, d'autres plus modérés questionnant le bien-fondé de son approche. Ainsi, l'intellectuel afroaméricain, Glenn Loury se permettait-il d'ironiser lorsque Lefkowitz affirme dans son ouvrage que les Afrocentristes « volent aux Grecs anciens et à leurs descendants l'héritage leur appartenant de droit ». Pour Loury, la démarche de Lefkowitz attribuant un héritage universel aux seuls descendants « biologiques » des Anciens apparaît essentialiste, au même titre que celle d'Afrocentristes qui cherchent à faire d'Hannibal, de Cléopâtre ou de Ramses II des Noirs, ancêtres des populations afro-américaines contemporaines (Moses $1998: 8$ ).

Plus importante à nos yeux, la critique formulée par Wilson Jeremiah Moses (1998) à l'égard de l'ouvrage aspirait à changer de regard sur l'Afrocentrisme. Comme après lui, Christine Chivallon (2004 : 122), Moses s'interrogeait sur la possibilité d'éviter l'écueil polémique dans une réflexion sur l'Afrocentrisme et appelait à s'extraire d'un débat, souvent contre-productif, opposant Afrocentristes à anti-Afrocentristes. Le tort de

1 En 2000, la revue Politique Africaine a dédié sa rubrique «Autour d'un livre » à deux ouvrages consacrés à 1'Afrocentrisme. Afrocentrismes. L'histoire des Africains entre Egypte et Amérique (2000) discuté ici et Afrocentrism. Mythical Past and Imagined Homes de Stephen Howe (1998). Participèrent au débat, Mohamed Mbodj, Jean Copans, Wim Van Binsbergen, François-Xavier Fauvelle-Aymar et Stephen Howe. Dans ce débat, plusieurs positions apparurent. Une critique virulente émanait des plumes de Stephen Howe, Fauvelle-Aymar et Jean Copans. De leur côté, Mohamed Mbodj et Wim Van Binsbergen se retrouvaient dans une position modérée faisant de l'Afrocentrisme une théorie qui, malgré ses défauts endémiques, fournit des hypothèses valables à reformuler scientifiquement. Ainsi, pour Van Binsbergen il est aujourd'hui nécessaire d'intégrer le point de vue afrocentriste dans les débats scientifiques plutôt que de le « reléguer au rang de fausse conscience » ( Mbodj, Copans, Van Binsbergen, Fauvelle-Aymar, Howe, 2000 : 177). 
Lefkowitz réside alors selon lui dans le fait qu'elle "s'intéresse davantage à prouver que Cléopâtre était une pure 'aryenne' qu'à analyser les circonstances qui ont produit le phénomène de l'Egyptocentrisme noir » (Moses 1998 : 3). Par là, Moses revendique la nécessité de replonger l'Afrocentrisme dans son contexte états-unien et afro-américain et de le considérer non pas comme une théorie scientifique contre laquelle s'insurger, à tort ou à raison, mais comme un objet construit historiquement dont il faut appréhender l'évolution et les différents avatars².

Avec Wilson Jeremiah Moses, l'Afrocentrisme devient donc un objet d'étude, au même titre que le nationalisme ou le messianisme auxquels il le relie, que l'historien se doit d'analyser. Son ouvrage, Afrotopia (1998), se donne pour but d'étudier la genèse du discours afrocentriste, en insistant sur ses multiples influences : le nationalisme noir bien sûr, mais aussi les nationalismes européens, le romantisme et l'anthropologie culturaliste. Dans la lignée des écrits de Wilson Jeremiah Moses, nous nous proposons dans ce dossier thématique de considérer l'Afrocentrisme non seulement comme un objet historique mais aussi anthropologique et sociologique. Nébuleuse, l'Afrocentrisme devient alors un objet aux multiples facettes, dont on peut analyser la construction et les métamorphoses ainsi que ses constantes manipulations opérées par une communauté afro-américaine qui, aux Etats-Unis, l'utilise à des fins politiques, thérapeutiques et organisationnelles. Dans cette logique, les articles constituant ce numéro thématique se concentreront sur la dimension états-unienne du phénomène afrocentriste, pays où ce courant d'idées est aujourd'hui le plus représenté et le plus puissant.

\section{Afrocentrisme entre philosophie de l'Histoire et nationalisme}

Il semble que le terme « afrocentrique » soit apparu pour la première fois sous la plume du sociologue afro-américain W.E.B. Du Bois en 1962. Invité par Kwame Nkrumah à Accra au Ghana dans le but d'y rédiger une encyclopédie sur les populations noires, l'Encyclopedia Africana, Du Bois insistait, dans un document non publié, sur son intention de diriger un volume « volontairement Afro-Centrique, mais prenant en compte l'impact du monde extérieur sur l'Afrique et l'impact de l'Afrique sur le monde extérieur » (in Moses 1998 : 2). Du Bois, important militant du panafricanisme, voyait dans son projet un moyen de donner la parole aux peuples d'Afrique, d'en faire des acteurs de leur propre Histoire au moment même où ceux-ci se lançaient dans la construction de leurs Etats nouvellement indépendants.

Lié chez Du Bois à un projet scientifique et politique destiné à donner la parole aux opprimés, le terme Afrocentric connaîtra ses heures de gloire à partir de la fin des années soixante. En réalité, l'histoire du mouvement afrocentriste aux Etats-Unis est indissociable de l'avènement de départements d'études dits ethniques dans les universités américaines, départements nés en pleine ère du Black Power, lorsqu'une jeunesse noire radicalisée se battait pour la prise en compte de son « expérience » au sein des cursus universitaires. Afrocentriques, au sens où Du Bois l'entendait, ces départements d'études African-American, Black ou Africana, se donnaient pour but d'inclure l'Histoire afroaméricaine dans le récit de l'Histoire états-unienne.

2 Dans une publication antérieure, j'ai montré comment l'absence de contextualisation du discours afrocentriste dans la littérature critique avait contribué à négliger les relations entre Afrocentrisme et Islam noir aux Etats-Unis (Guedj 2003). 
A partir des années 1980, l'Afrocentrisme académique entra dans une nouvelle phase avec les publications d'Arthur Lee Smith, plus connu sous le nom de Molefi Asante (Asante 1987, 1988, 1990). Celui-ci tendait à définir l'Afrocentrisme comme une théorie cherchant à remettre l'Afrique au cœur de l'Histoire de l'humanité. Toutefois, ses principaux écrits associèrent à l'Afrocentric duboisien tout un appareil conceptuel et idéologique, grandement hérité des écrits de l'historien sénégalais Cheikh Anta Diop.

A partir de 1990, la pensée d'Asante évolua peu de sorte qu'il est aisé, avec Stephen Howe (1998), de la résumer en une série de points précis. Le premier cheval de bataille de la théorie afrocentriste, telle qu'elle est définie par Asante, revendique le fait que l'humanité se serait d'abord développée en Afrique avant de se répandre en Asie, aux Amériques puis en Europe. Les Africains entretiendraient avec le reste des habitants de la terre un rapport de primordialité chronologique et ce, particulièrement avec les Européens, « race » relativement jeune dans l'histoire de l'humanité. Des découvertes archéologiques comme celles des squelettes de Lucie et de l'Eve africaine constituent, pour l'auteur, des témoignages d'une telle antériorité.

Le second point défendu par Asante est que la première civilisation mondiale aurait pris place en Egypte ancienne ou Kemet. Cette civilisation était «noire » et ses ingénieurs provenaient surtout de la partie sud du pays, Koush, laquelle s'apparente au Soudan actuel. L'étude des phénotypes égyptiens tels qu'ils sont visibles sur les papyrus ou les fresques des temples et des tombes apporterait la preuve de la négritude de cette population, et nombreux sont les disciples d'Asante qui ont fait de cette recherche leur spécialité.

Ensuite, Asante insiste fréquemment sur le fait que le rayonnement de la civilisation égyptienne s'est étendu sur la totalité du continent noir. Il étudie ce qu'il considère comme des points communs entre la langue égyptienne et un certain nombre de langues africaines comme le wolof ou le haussa. Pour lui, le chercheur afrocentriste serait à même de mettre à jour les liens culturels qui unissent les populations africaines à la civilisation et aux mœurs de l'Egypte antique et la linguistique en constituerait une preuve évidente.

De plus, selon Asante, la culture égyptienne se serait également diffusée au Nord, jusqu'à constituer la source d'inspiration première des civilisations qui apparurent plus tardivement en Grèce et à Rome d'abord puis partout en Europe. Athéna était, pour Asante, une déesse noire et les Grecs, des usurpateurs ayant volé honteusement leurs traditions aux Africains d'Egypte et de Nubie. Ces propos d'Asante trouveront un écho favorable dans l'ouvrage de Martin Bernal Black Athena (1987), rédigé par un historien blanc, aujourd'hui considéré comme un tenant des théories afrocentristes.

Enfin, pour Asante, comme le défendait également Cheikh Anta Diop (1959), les traditions africaines constituent autant de manifestations d'une culture unique. Depuis son foyer égyptien, la culture africaine, au singulier, s'est diffusée dans la totalité du continent. Les frontières nationales et culturelles en Afrique sont le fruit de la colonisation et des efforts forcenés des Européens pour détruire cette unité, menace potentielle à leur hégémonie. Ce faisant, l'ensemble des populations noires forme une nation unitaire, avec ses propres croyances, valeurs et pratiques sociales (Howe 1998 : 231).

Chez Asante, cette notion de l'unité culturelle des peuples noirs dépasse toutefois largement le simple continent africain pour inclure dans cet ensemble les populations dites de la « diaspora ». Pour l'auteur, les Afro-Américains des Etats-Unis, les Noirs du Brésil et de la Guadeloupe sont tous pétris de cette culture africaine et chacun de leurs actes témoigne de leur africanité. La nation noire, dans ses formes afro-caribéennes, africaines et afro-américaines est caractérisée par ce qu'Asante appelle « les rythmes de l'unité », 
autant de variations coutumières que tout ramène à une culture unique « territorialisée " en Egypte antique (Asante et Asante 1985 : X).

Autour de Molefi Asante et de sa doctrine afrocentriste, se sont progressivement rassemblés de nombreux intellectuels. A la tête du département d'études africainesaméricaines de l'université de Temple à Philadelphie, Asante a su recruter des chercheurs souvent formés par ses soins et défendant corps et âme les présupposés de son Afrocentrisme. Par ailleurs, des auteurs parfois intellectuellement éloignés d'Asante se sont trouvés réappropriés par les Afrocentristes, utilisés pour mettre en place leur récits historiques, parmi ceux-ci Cheikh Anta Diop, entre autres pour ses travaux sur l'Egypte antique, Melville Herskovits, pour sa quête des survivances africaines dans la culture afro-américaine et ses démonstrations sur l'unité culturelle de l'Afrique noire, et plus récemment Martin Bernal, auteur du très controversé Black Athena.

Toutefois, la littérature spécialisée sur la question tend à dégager à l'intérieur de cet ensemble afrocentrique plusieurs écoles, plusieurs tendances, voire même plusieurs Afrocentrismes. En 1999, comme nous le rappelle Christine Chivallon (2004 : 123), l'article «Afrocentrisme » rédigé par Robert Fay (1999: 45) dans l'Encyclopedia Africana de Kwame Anthony Appiah et Henry Louis Gates distingue une branche dite « modérée » incarnée par Asante et ses disciples et une branche « dure », parfois ouvertement raciste et cherchant à prouver par divers moyens la supériorité de la « race » noire sur la blanche.

Une même distinction se retrouve dans les écrits d'Algernon Austin (2006 : 119) qui oppose l'université afrocentrique incarnée par Asante à ce qu'il appelle les théories de la mélanine, ouvertement biologistes et essentialistes. Parmi ces " théoriciens de la mélanine », Frances Cress Welsing $(1991)^{3}$, psychiatre afro-américaine défend l'idée selon laquelle la « race » blanche serait génétiquement inférieure d'où découleraient chez ses membres d'importants troubles psychologiques, qu'ils compenseraient en tentant d'imposer leur suprématie sur l'humanité4. Populaires, revendiquées par de nombreux chercheurs et enseignants d'université, tels Carol Barnes, T. Owens Moore et Leonard Jeffries, les théories de la mélanine sont depuis 1987, le sujet de conférences annuelles où l'on tente de prouver que la « mélanine est un liquide sédatif permettant à l'être humain de rester calme, relaxé, dévoué et civilisé » (Barnes in Austin 2006 : 120).

Ces deux composantes de l'Afrocentrisme, telles qu'elles sont dégagées par Algernon Austin (2006) et Robert Fay (1999 : 45) semblent en effet s'opposer sur de nombreux points. Dans une même logique, Jean Copans (voir Mbodj, Copans et al. 2000 : 169) propose de ne pas évoquer l'Afrocentrisme au singulier mais d'apposer à ce terme le « S » du pluriel, signifiant ainsi la multiplicité de ses avatars. Toutefois, malgré la diversité des formes prises par l'Afrocentrisme, il semble que l'ensemble de ses penseurs se retrouve dans l'orientation politique qu'ils donnent à leurs écrits et en particulier dans la centralité accordée, même dans le cas de Molefi Asante, à la dimension raciale.

En effet, l'entreprise d'Asante s'accompagne dès Afrocentricity en 1988 de l'établissement d'un programme politique et thérapeutique dont la visée explicite est de

3 Frances Cress Welsing est par ailleurs bien connue pour ses propos antisémites. Elle est justement critiquée par Stephen Howe dans son ouvrage sur l'Afrocentrisme, voir Howe (1998 : 285).

4 Pour une réflexion sur les théoriciens de la mélanine tels Frances Cress Welsing, T. Owens Moore et Carol Barnes, voir le sous-chapitre « The Negro is only Biological» de l'ouvrage de Paul Gilroy, Against Race. Imagining Political Culture Beyond the Color Line (2000 : 254-265). 
restaurer la fierté au sein des consciences noires. En ce sens, Asante tout comme Barnes et Cress Welsing est l'auteur d'une vaste doctrine nationaliste dont l'objectif premier est la rédaction d'une Histoire, la recollection d'un héritage et la création d'une tradition commune, à même de rassembler l'ensemble des populations noires de la planète, dans une entité unique, une Nation panafricaine et transnationale. "Communauté imaginée », comme nous le rappelle Benedict Anderson (1983), la Nation ainsi créée par les Afrocentristes s'appuie sur un passé glorieux, en permanente reconstruction, revendiqué dans une logique « contributionniste $»^{5}$ (Patterson 1971) comme un apport essentiel à l'histoire de l'humanité. Autant de traditions inventées, " tentant d'établir une continuité avec un passé historique approprié » (Hobsbawm $2006: 12)^{6}$. Les intellectuels afrocentristes en effet se livrent à de véritables « exercices d'ingénierie sociale » (ibid.) faisant des Noirs une Nation n'ayant pas souffert de la rupture fondatrice causée par l'expérience traumatique de l'esclavage.

Toutefois, la Nation afrocentriste n'existe pas uniquement sur la base d'un passé réinventé. Elle est aussi, pour reprendre les termes d'Ernest Renan (1947), une entité dont «l'essence est que tous les individus (la formant) aient beaucoup de choses en commun ». Or, c'est dans l'appartenance à la « race » noire qu'Asante et ses disciples trouvent le terreau fertile d'une solidarité horizontale et communautaire. La « race », comme ce fut le cas dans l'ensemble des nationalismes afro-américains états-uniens depuis le $19^{\mathrm{e}}$ siècle, est ici un ciment de la Nation imaginée, un « liant » qui, au même titre que l'Histoire, rassemblerait dans une même communauté les Africains et les Afro-Américains. Ainsi, s'établit une adéquation entre «Africain » et « race noire » (Chivallon $2006: 51$ ) permettant d'expliquer dans le contexte états-unien, l'obsession d'Asante pour la figure d'une Cléopâtre à la peau pigmentée et la quête effrénée de preuves biologiques d'une supériorité de la race noire chez les théoriciens de la branche dure de 1'Afrocentrisme ${ }^{7}$.

\section{Afrocentrisme et pratiques sociales}

Chez Molefi Asante, la création de cette Nation noire imaginée s'appuie alors sur un réel programme dont la clé de voûte est ce qu'il appelle l' «Afrocentricité ». L'Afrocentricité, en tant que doctrine, s'adresse à l'ensemble des populations noires et suppose, comme le rappelait Asante lui-même lors d'une conférence organisée à Paris le 31 octobre 2006 par l'associationAfricamaat ${ }^{8}$, de se recentrer sur sa propre expérience, d'adopter un mode de vie conforme à son essence et ses supposées prédispositions « noires ». Là où l'Afrocentrisme défini par Asante reposait sur une philosophie de l'Histoire, l'Afrocentricité, telle qu'il la décrit, est un art de vivre, la capacité pour les Noirs

5 Orlando Patterson (1971) appelle « contributionnisme », la revendication chez les nationalistes afroaméricains d'un passé glorieux témoignant de la contribution des Noirs à l'Histoire de l'humanité et à l'évolution humaine.

6 Pour une réflexion théorique récente sur le paradigme de traditions inventées, voir Otto et Pedersen (2005).

7 Notons toutefois qu'il existe dans les théories du nationalisme noir, une nuance entre l'utilisation des termes « Noirs » et « Africains » même s’ils désignent en fait une même « réalité ». Pour les membres du mouvement « akan », par exemple, dont il est question dans l'un des articles de ce numéro, se revendiquer comme Africain c'est introduire une dimension territoriale aux origines que ne permet pas la simple identification à la « race » noire.

8 La diffusion des idées afrocentristes en France prend actuellement une ampleur importante. 
de refaire corps avec leur essence et leurs déterminismes jugés premiers. L'Afrocentrisme universitaire d'Asante s'accompagne donc de pratiques sociales et culturelles regroupées sous le terme «Afrocentricité », autant de postures que l'Afro-Américain doit adopter s'il veut être un Noir ou un Africain « authentique ».

Or, derrière cette défense de l'Afrocentricité chez Asante, on retrouve en réalité les traces du nationalisme culturel, qui depuis les années soixante, se déploie dans certains milieux militants afro-américains. En effet, depuis la fin des années cinquante, l'Afrique est redevenue une source positive d'identification pour les Noirs américains. Alors que dans les années 1930, la Nation of Islam tentait d'éviter tout rapprochement symbolique entre les Noirs des Etats-Unis et des Africains jugés primitifs et sauvages, en faisant de ses fidèles des Afro-Asiatiques descendants d'une «tribu » originelle et divine provenant de La Mecque, les années soixante honoreront le continent noir, continent en voie de décolonisation et nouvelle inspiration possible pour des communautés luttant sur le territoire états-unien contre la ségrégation et l'oppression'.

Rapidement, cette vogue de l'Afrique se fit ressentir dans plusieurs sphères de la vie afro-américaine. Dans les rues de Harlem à New York ou du Watts à Los Angeles, certains Afro-Américains optèrent pour des vêtements considérés comme " africains », adoptèrent de nouvelles coupes de cheveux, dites « afro », naturelles, et changèrent parfois leur identité pour s'approprier des noms jugés eux aussi originaires de la «Terre Mère » (Asante, Karenga, Oseijeman, Dinizulu...). A l'intérieur du champ religieux afro-américain, naquirent également de nouvelles pratiques, empruntées aux Yoruba du Nigeria $^{10}$, aux Akan du Ghana ${ }^{11}$ voire à l'Antiquité égyptienne, pratiques considérées comme autant de manifestations dans le Nouveau Monde d'une Afrique jugée authentique.

Par ailleurs, nombreux furent les artistes populaires pour qui, à l'époque, le continent noir devint une préoccupation. Ainsi, alors que John Guillermin n'hésita pas à tourner en Afrique un nouvel opus de Shaft, intitulé Shaft in Africa, plusieurs jazzmen cherchèrent dans le continent noir des sources d'innovation pour leur art. Ifé de Miles Davis, Juju de Wayne Shorter, Ogunde de John Coltrane, sans parler des prestations des membres de l'Art Ensemble of Chicago en boubou et batakari, constituent autant d'exemples de cet intérêt renouvelé des Noirs pour la «Terre Mère $»^{12}$.

Pour ces créateurs afro-américains tout comme pour ces militants pratiquant des religions dites « africaines » et optant pour des vêtements originaires du continent noir, l'art, la culture devenaient de réels projets politiques qui pourraient leur permettre de revendiquer leur connexion avec la «Terre Mère » et d'exprimer clairement leur solidarité avec les populations africaines. Le militant noir se devait donc d'être le symbole de cet Afro-Américain, qui cherche en Afrique une inspiration pour son propre combat. Nous le voyons, l'engouement pour le continent noir, si déterminant dans les années soixante,

9 Sur le passage aux Etats-Unis d'un nationalisme musulman centré sur la figure de l'Afro-Asiatique à un nationalisme culturel « afrocentré », voir Guedj (2006 : 67-95) ou Meriwether (2002).

10 Sur la pratique de la religion « yoruba » aux Etats-Unis, voir, entre autres, Capone (2005) et Hunt (1979).

11 Voir Guedj (2006).

12 Cet engouement toucha également des artistes majeurs de la scène soul et funk. En 1971, fut organisé à Accra, en commémoration de l'indépendance du Ghana, un concert historique intitulé Soul to Soul réunissant des musiciens afro-américains comme Wilson Pickett, Ike et Tina Turner, The Staple Singers et des artistes locaux tels Kwa Mensah. 
décliné dans plusieurs pratiques sociales afro-américaines, est en réalité le résultat d'une quête culturelle, à travers laquelle des Noirs américains tentèrent de refaire corps avec leur supposée identité d'avant l'esclavage, identité africaine, qui, pour les militants concernés, est conçue comme vivante bien qu'endormie, mise entre parenthèses, dans le for intérieur des Afro-Américains.

Autour de leaders, tels Maulana Karenga et Amiri Baraka, le nationalisme culturel proposa à ses adeptes de se lancer dans une recherche personnelle de leurs racines africaines. Pour ces penseurs, recouvrer son identité première revenait à vivre conformément à son essence et à se séparer, psychologiquement, - ou mentalement, dirait Amiri Baraka, de l'Amérique blanche environnante. Séparatisme symbolique et culturel, ce nationalisme se construisit sur une conception de la culture, profondément états-unienne, présente aussi bien dans les idéologies du melting pot et du multiculturalisme que dans l'anthropologie culturaliste, selon laquelle chaque individu est entièrement modelé (patterned) par la culture à laquelle il appartient; une culture dont il est la création. Chez Karenga comme chez Asante, refaire corps avec sa culture africaine revient alors à recouvrer son essence première et noire.

Acteurs et héritiers du nationalisme culturel, Asante et ses disciples sont eux aussi de fervents partisans de ces pratiques culturelles et sociales visant à retrouver les racines de l'Afrique. Leur Afrocentrisme s'accompagne d'actes, de pratiques destinés à replacer une culture africaine réinventée au cœur de la culture noire américaine. Ce faisant, l'Afrocentrisme, avec ses multiples facettes, se dévoile bien plus complexe qu'une théorie scandaleuse inculquée aux étudiants de certains départements d'études africaines-américaines. A l'image du panafricanisme, il est le résultat de constructions à plusieurs niveaux, intellectuelles, politiques, sociales et/ou culturelles.

En 1962, George Shepperson proposait une définition du panafricanisme en deux volets. Le Panafricanisme avec un $\mathrm{P}$ majuscule désignerait les activités politiques d'intellectuels tels W.E.B. Du Bois ou George Padmore, lesquels cherchaient à établir des liens diplomatiques entre différentes communautés noires de la planète. Le panafricanisme, annoncé par la minuscule, recouvrerait lui, des actions quotidiennes, routinières, telles celles d'un écrivain de la Négritude qui défend son point de vue « noir », d'un musicien qui s'inspire et mélange les influences du continent et de ce que l'on appelle parfois aujourd'hui la « diaspora », ou d'un Afro-Américain, qui, participant aux réunions de l'UNIA de Marcus Garvey, chante l'hymne du mouvement Ethiopia, thou land of our fathers. Cette distinction, Shepperson (1962) l'évoque bien, ne dégage pas, bien sûr, deux catégories hermétiques. De nombreux éléments constituant le Panafricanisme avec une majuscule relèvent également du panafricanisme avec une minuscule. La dichotomie proposée par Shepperson fut parfois critiquée par les spécialistes de la question ${ }^{13}$. Toutefois, elle eut l'avantage non négligeable de mettre en avant la dimension culturelle de toute doctrine politique et intellectuelle.

En s'inspirant du principe de la dichotomie Majuscule/minuscule établi par Shepperson, on pourrait alors distinguer un Afrocentrisme académique, intellectuel, philosophie de l'Histoire cherchant à remettre l'Afrique au cœur de l'Histoire de l'humanité, tant

13 Winston James (2004 : 126) regrette que l'emploi de la majuscule et de la minuscule dans la classification de Shepperson (1962) puisse induire une hiérarchie contre-productive entre les deux composantes dégagées. Pour une discussion autour de la définition du terme panafricanisme, voir Bonacci (2008 : 52). 
critiquée par les anti-Afrocentristes, et des afrocentrismes, avec une minuscule et ici au pluriel, autant de pratiques tentant de recréer aux Etats-Unis une Afrique noire rêvée, constamment réinventée et imaginée comme « pure » et « authentique ». Mais il est clair que les afrocentrismes ne cessent d'alimenter les débats académiques, et que ces débats interviennent à leur tour dans l'invention de l'Afrique idéale recréée aux Etats-Unis.

\section{Afrocentrismes américains}

Pour chacun des auteurs de ce dossier thématique, le défi a toujours été de rattacher la théorie afrocentriste à son contexte socio-culturel et d'insister sur les passerelles constamment établies entre doctrine et pratiques sociales et/ou culturelles. Ce faisant, les auteurs de ce dossier ont tenté d'apporter un regard anthropologique, historique et sociologique sur les dynamiques observées en analysant les processus de construction et de réappropriation de l'Afrique par les Noirs des Etats-Unis à plusieurs époques et dans plusieurs sphères de la vie sociale.

La première partie de ce numéro s'intéresse aux esthétiques et aux pratiques artistiques afrocentristes, pratiques et discours directement issus de la révolution culturelle des années soixante. L'article de Kristin L. Ellsworth revient sur les mouvements artistiques des années soixante qui firent de l'Afrique leur principale source d'inspiration. L'auteur analyse, en s'intéressant aux œuvres des artistes du collectif Africobra, l'élaboration d'un afrocentrisme, qu'elle nomme « visuel » alliant toujours symbolisme et militantisme. Dans cet article, l'art est décrit comme un acte performatif, dans lequel le créateur s'engage pour lutter contre le racisme et l'oppression.

Dans sa contribution, Sarah Fila-Bakabadio se concentre, quant à elle, sur la diffusion aux Etats-Unis de tissus africains et en particulier du kente ghanéen. En insistant sur les liens historiques reliant Afro-Amérique et Afrique, elle analyse les processus de construction d'une africanité américaine à travers l'évolution des représentations du kente au sein de la communauté noire. Du nationalisme afro-américain de la première moitié du vingtième siècle au militantisme des années 1980 et 1990, elle montre comment le kente est devenu un outil intervenant dans les logiques de réafricanisation de la culture noire. Ce faisant, le kente rejoint dans les milieux militants et populaires le patchwork, quilt, autrefois emblème de l'artisanat noir américain.

Enfin, Bénédicte Monville De Cecco interroge les relations entre Afrocentrisme et littérature à travers une réflexion à la fois sur les écrits de l'auteur afro-américain John Edgar Wideman et sur les critiques de son œuvre émanant d'intellectuels apparentés au courant afrocentriste. Dans son article, elle montre comment, contrairement à ce que laissent supposer de nombreux textes d'analyse produits sur son œuvre, John Edgar Wideman se dissocie de l'approche afrocentriste par sa démarche anti-essentialiste. Ainsi, Wideman se fait l'interprète d'une culture afro-américaine aux référents et aux mémoires multiples alliant Amérique, imaginaires africains et négritude.

La seconde partie de ce dossier se propose d'analyser les relations entre A/afrocentrismes et politique. Dans ma contribution, j'étudie les processus de construction d'une identité « africaine » aux Etats-Unis au sein d'un mouvement politico/religieux, le mouvement « akan ». En analysant les rituels mis en place par les pratiquants de la religion « akan », je démontre comment l'invention d'une Afrique reterritorialisée aux Etats-Unis donne lieu à des processus d'identification multiples 
et complexes passant par une renégociation des notions de panafricanisme et d'afroaméricanité.

Dans une même logique, John L. Jackson s'interroge, dans son article, sur la revendication d'une identité hébraïque au sein de certains mouvements nationalistes afroaméricains. En relatant de récentes enquêtes de terrain menées en Israël et aux Etats-Unis, il propose de reconsidérer les liens imaginés par les adeptes afro-américains entre judaïté, Afrique et A/afrocentrisme(s). Ancrant son étude dans une analyse des traitements du corps et des processus de purification en œuvre dans les logiques de conversion aux mouvements, l'auteur nous invite à reconsidérer les limites de l'Afrique afrocentriste, une Afrique dont les représentants pourraient faire remonter leur origine autant aux pharaons de l'Egypte qu'au peuple hébraïque de la Bible.

Enfin, le dernier article de ce dossier, rédigé par Algernon Austin s'intéresse à un phénomène hautement contemporain. A partir de plusieurs textes rédigés par Barack Obama dont son autobiographie, Dreams of my father (2004), Austin étudie les représentations de l'Afrique incarnées par le président et s'interroge sur les relations entretenues par Obama avec le courant afrocentriste et en particulier avec le pasteur Jeremiah A. Wright, Jr, leader de l'Eglise controversée qu'il fréquentait jusqu' au moment de sa campagne. Or, si pour Algernon Austin, Obama n'opte jamais pour une vision afrocentriste de ses origines africaines, l'auteur reconnaît chez le président l'influence de postures politiques qu'il identifie à une " ère afrocentrique », période de l'histoire du nationalisme noir où le militantisme, se dissociant Black Power, tendait à devenir moins radical.

S'intéressant à des pratiques artistiques, intellectuelles, religieuses et politiques, ce numéro thématique dresse un portrait du kaléidoscope afrocentriste dans sa diversité et sa multiplicité mais aussi dans ses continuités et ses cohérences transversales. Ce faisant, il propose une réflexion sur les processus d'identification élaborés par des acteurs afroaméricains pour lesquels l'affirmation et la construction d'un passé africain permettent de revendiquer une place légitime dans une Amérique dite multiculturelle.

\section{Références citées}

Anderson, Benedict, 1983. Imagined Communities. Reflections on the Origin and Spread of Nationalism. Londres et New York : Verso.

Asante, Molefi,

1987. The Afrocentric Idea. Philadelphie : Temple University Press.

1988. Afrocentricity. Trenton : Third World Press.

1990. Afrocentricity and Knowledge. Trenton : Africa World Press.

Asante, Molefi Kete et Kariamu Welsh Asante,1985. African Culture. The Rythms of Unity. Trenton : African World Press.

Austin, Algernon, 2006. Achieving Blackness. Race, Black Nationalism and Afrocentrism in the Twentieth Century. New York et Londres : New York University.

BERnAL, Martin, 1987. Black Athena. The Afrocentric Roots of Classical Civilization, vol. I : The Fabrication of Ancient Greece, 1785-1985, vol. II : The Archeological and Documentary Evidence. New Brunswick : Rutgers University Press.

Bonacci, Giulia, 2008. Exodus ! L'histoire du retour des Rastafariens en Ethiopie. Paris : Scali. 


\section{Afrocentrismes américains}

CAPOnE, Stefania, 2005. Les Yoruba du Nouveau Monde. Religion, ethnicité et nationalisme noir aux Etats-Unis. Paris : Karthala.

Chivallon, Christine,

2004. La diaspora noire des Amériques. Expériences et théories à partir de la Caraïbe. Paris : CNRS éditions.

2006. « Diaspora noire des Amériques : une réflexion conduite à partir de la notion de lien transétatique », Autrepart, 38, pp. 39-61.

Cress Welsing, Frances,1991. The Isis (Yissis) Papers. The Key to the Colors. Chicago : Third World Press.

Diop, Cheikh Anta,

1954. Nations nègres et culture. Paris : Présence africaine.

1959. L'unité culturelle de l'Afrique noire. Paris : Présence africaine.

1967. Antériorité des civilisations : Mythes ou vérités historiques. Paris : Présence africaine.

Fauvelle-Aymar, François-Xavier, Jean-Pierre Chrétien et Claude-Hélène Perrot (éds), 2000. Afrocentrismes. L'histoire des Africains entre Egypte et Amérique. Paris : Karthala.

FAY, Robert, 1999. «Afrocentrism » in Appiah, Kwame Anthony et Gates, Henry Louis, Encyclopedia Africana. New York : Basic Civitas Books.

Gilroy, Paul, 2000. Against Race. Imagining Political Culture Beyond the Color Line. Cambridge : Belknap Press of Harvard University Press.

Guedu, Pauline,

2003. «Des 'Afro-Asiatiques' et des 'Africains': islam et afrocentrisme aux Etats-Unis », Cahiers d'études africaines, XLIII (4), 172, pp. 739-760.

2006. Le Chemin du Sankofa. Religion et Identité « akan » aux Etats-Unis, Thèse de doctorat, Université Paris X Nanterre.

HobsBawm, Eric, 2006. «Introduction » in Hobsbawm, Eric et Ranger, Terence (éds), L'invention de la tradition. Paris : Editions Amsterdam, pp. 1-14.

Howe, Stephen, 1998. Afrocentrism : Mythical Pasts and Imagined Homes. Londres et New York : Verso.

Hunt, Carl Monroe, 1979. The Yoruba Movement in America. Washington D.C. : University Press of America.

JAMES, Winston, 2004. «The Wings of Ethiopia. The Caribbean Diaspora and Pan-African Projects from John Brown Russwurm to George Padmore », in G. Fabre et K. Benesch (éds), African Diasporas in the New and Old Worlds. Consciousness and Imagination. Amsterdam et New York : Rodopi, pp. 121-157.

Lefkowitz, Mary, 1996. Not Out of Africa. How Afrocentrism Became an Excuse to Teach Myth As History. New York : Basic Books.

Lefkowitz, Mary et G.M. Rogers (éds), 1996. Black Athena Revisited. Chapel Hill : University of North Carolina Press.

Mbodj, Mohamed, Jean Copans, Wim Van Binsbergen, François-Xavier Fauvelle-Aymar, Stephen Howe, 2000. «Autour d'un livre, Afrocentrismes. L'histoire des Africains entre Egypte et Amérique de JeanPierre Chrétien, François-Xavier Fauvelle-Aymar et Claude-Hélène Perrot (dir.) », Politique Africaine, 79, octobre 2000, pp. 165-191.

Meriwether, James, 2002. Proudly We Can be African. Black Americans and Africa 1935-1961. Durham : University of North Carolina Press.

Moses, Wilson Jeremiah, 1998. Afrotopia. The Roots of African American Popular History. Cambridge : Cambridge University Press.

Oвама, Barack, 2004. Dreams of My Father : A Story of Race and Inheritance. New York : Three Rivers Press.

OBenga, Théophile, 2001. Le sens de la lutte contre l'africanisme eurocentriste. Gif-sur-Yvette : L'Harmattan, Khepera. 
Ortiz de Montellano, Bernard, 2000. « Black Warrior dynasts. L'afrocentrisme et le Nouveau Monde » in Fauvelle-Aymar, François-Xavier, Chrétien, Jean-Pierre et Perrot, Claude-Hélène (éds), Afrocentrismes. L'histoire des Africains entre Egypte et Amérique. Paris : Karthala, pp. 249-270.

Otto, Ton et Poul Pedersen, 2005. « Disentangling Traditions. Culture, Agency and Power », in Otto, Ton et Pedersen, Poul (éds), Tradition and Agency. Tracing Cultural Continuity and Invention. Aarhus : Aarhus University Press, pp. 11-49.

Owens Moore, T., 1995. The Science of Melanin. Dispelling the Myths. Silver Spring : Venture Book.

Patterson, Orlando, 1971. « Rethinking Black History », Harvard Educational Review, 3, pp. 297-315.

Renan, Ernest, 1947. «Qu'est ce qu'une nation ? » in Oeuvres Complètes. Paris : Calman Lévy, vol. 1, pp. 887-906.

ShePperson, George, 1962. «'Pan-Africanism' and 'pan-africanism' : Some Historical Notes », Phyllon XXIII/4, pp. 346-358.

Walker, Clarence, 2000. « Les a posteriori de Molefi K. Asante » in François-Xavier Fauvelle-Aymar, Jean-Pierre Chrétien et Claude-Hélène Perrot (éds), Afrocentrismes. L’histoire des Africains entre Egypte et Amérique. Paris : Karthala, pp. 65-78. 